\title{
Recualificación del paisaje urbano del Bajo Augusta, São Paulo, Brasil. Conexiones vivas y movilidad sostenible.
}

\author{
Deize Sbarai Sanches Ximenes ${ }^{1}$, Denise Gonçalves Lima Malheiros ${ }^{2}$, \\ Fábio Cesar Moreira Manente ${ }^{3}$ \\ ${ }^{123}$ Departamento de Arquitetura e Urbanismo, Universidade Paulista - Campus Alphaville, \\ Universidade de São Paulo, São Paulo, Brasil, ${ }^{1}$ IEA USP Instituto de Estudos Avançados da \\ Universidade de São Paulo - Programa Cidades Globais, São Paulo, Brasil. \\ E-mail: ${ }^{1}$ deizesanches@usp.br, ${ }^{2}$ denisemalheiros@uol.com.br, ${ }^{3} \mathrm{fcmanente@gmail.com}$
}

\begin{abstract}
Resumen. El crecimiento urbano desordenado es una realidad en las ciudades brasileñas, agravado por la especulación inmobiliaria, que genera descalificación de los espacios públicos y vaciamiento de los centros urbanos. La calle es un organismo vivo, un espacio de apropiación pública, donde su uso puede mejorar o degradar la calidad de vida de las personas. El Bajo Augusta, área cortada por la calle Augusta, de la Avenida Paulista a la Plaza Roosevelt en el centro de São Paulo, símbolo de la ocupación urbana paulistana y escenario de manifestaciones culturales y sociales desde 1950, sufrió profundas transformaciones urbanas de degeneración del paisaje urbano, reflejo del rápido crecimiento económico y la descentralización provocada por inversiones de capital privado y de recursos de infraestructura pública, creando nuevos puntos de centralidad. La investigación objetiva la recalificación del paisaje urbano del Bajo Augusta basada en cinco ejes estructuradores: accesibilidad urbana, movilidad sostenible, identidad cultural, vivienda social y preservación ambiental; y en la legislación urbana vigente. Para que esta área sea eficiente $y$ accesible, nuevos escenarios urbanos se propusieron con conexiones vivas entre personas, lugares y actividades - valorización de la identidad cultural, uso compacto y diversificado, y nueva movilidad sostenible; un transporte de masa compacto a la demanda local y conectado a los diferentes modales, reduciendo la necesidad de desplazamientos. Esta propuesta pretende establecer espacios públicos llenos de vitalidad, nuevas oportunidades de trabajo, ocio y cultura, privilegiando la escala humana, regenerar las cualidades socioambientales y colaborar con el desarrollo sostenible del municipio de São Paulo.
\end{abstract}

Palabras clave: Recalificación urbana, espacio público, conexiones vivas, paisaje urbano y movilidad sostenible.

\section{Introducción}

De acuerdo con Jacobs (1961) la calle es un organismo vivo, un espacio de apropiación pública, donde su uso puede mejorar o degradar la calidad de vida de una ciudad. Por lo tanto, se comprende que la calificación de los espacios públicos y la apropiación de los vacíos urbanos van a privilegiar al peatón y regenerar las cualidades socioambientales. Así como, para que estos núcleos sean eficientes necesitan una movilidad; un transporte de masa compacto a la demanda local, y deben ser analizados a partir de la teoría de la ciudad compacta (ROGERS, 1997), donde los núcleos de uso mixto y compactos reducen las necesidades de desplazamientos y crean barrios sostenibles, llenos de vitalidad.

Al analizar el núcleo central de la ciudad de São Paulo, a partir de finales de la década de 1960 y durante los años 1970, se percibe un considerable vaciamiento, consecuencia de un 
momento de intenso crecimiento económico, grandes inversiones públicas en infraestructura y la formación de nuevas centrales.

El Bajo Augusta, área de la calle Augusta, ubicado en el centro de São Paulo, símbolo de la ocupación urbana paulistana y escenario de manifestaciones culturales y sociales desde la década de 1950, también sufre profundas transformaciones urbanas de degeneración del paisaje urbano y de los espacios públicos, reflejo de la descentralización y de un crecimiento difuso.

El panorama de la década de 1950, caracterizada por un fuerte sentimiento progresista, reflejó no sólo en la economía, sino también en la cultura de la nación como un todo (CANO, 2007), y la calle Augusta emerge como polo condensador de jóvenes de la época, pues era allí donde se concentraban los bares, cafeterías y espacios culturales como cines y teatros. Además de reducto joven, también era un polo de lujo RAGAZZO (2005) y la coloca como "Pasarela de la Gloria".

Conforme a ZIMMERMANN (2011) se entiende que el desarrollo de la calle Augusta como polo cultural fue relacionado con la emergencia de la Avenida Paulista como una nueva centralidad, donde las directrices de las nuevas legislaciones de uso y ocupación del suelo, incentivaron la especulación inmobiliaria y la verticalización de los edificios de oficinas. Otro factor que cooperó para el vaciamiento del lado central de la calle Augusta, conforme VILLAÇA (2001) y FRUGOLI JR, (1995) fue el surgimiento de las grandes empresas que necesitaban una nueva tipología de edificio en la década de 1960. Esa razón llevó al crecimiento en el centro de la ciudad era restrictivo debido al gran número de edificios históricos y al poco espacio vacío para la construcción de esos nuevos emprendimientos.

En 1990, la prostitución y las actividades ilícitas se tornaron más nítidas y usuales en la región del Bajo Augusta, creando una especie de atmósfera underground que varios artistas de la época retrataron, a ejemplo de, Ozunaldo Candeias, que en 1987 describe la ciudad como un todo un lugar de degradación humana a través de su película "Las Bellas de Billings" (EL ESTADO DE S. PAULO, 13/02/1987).

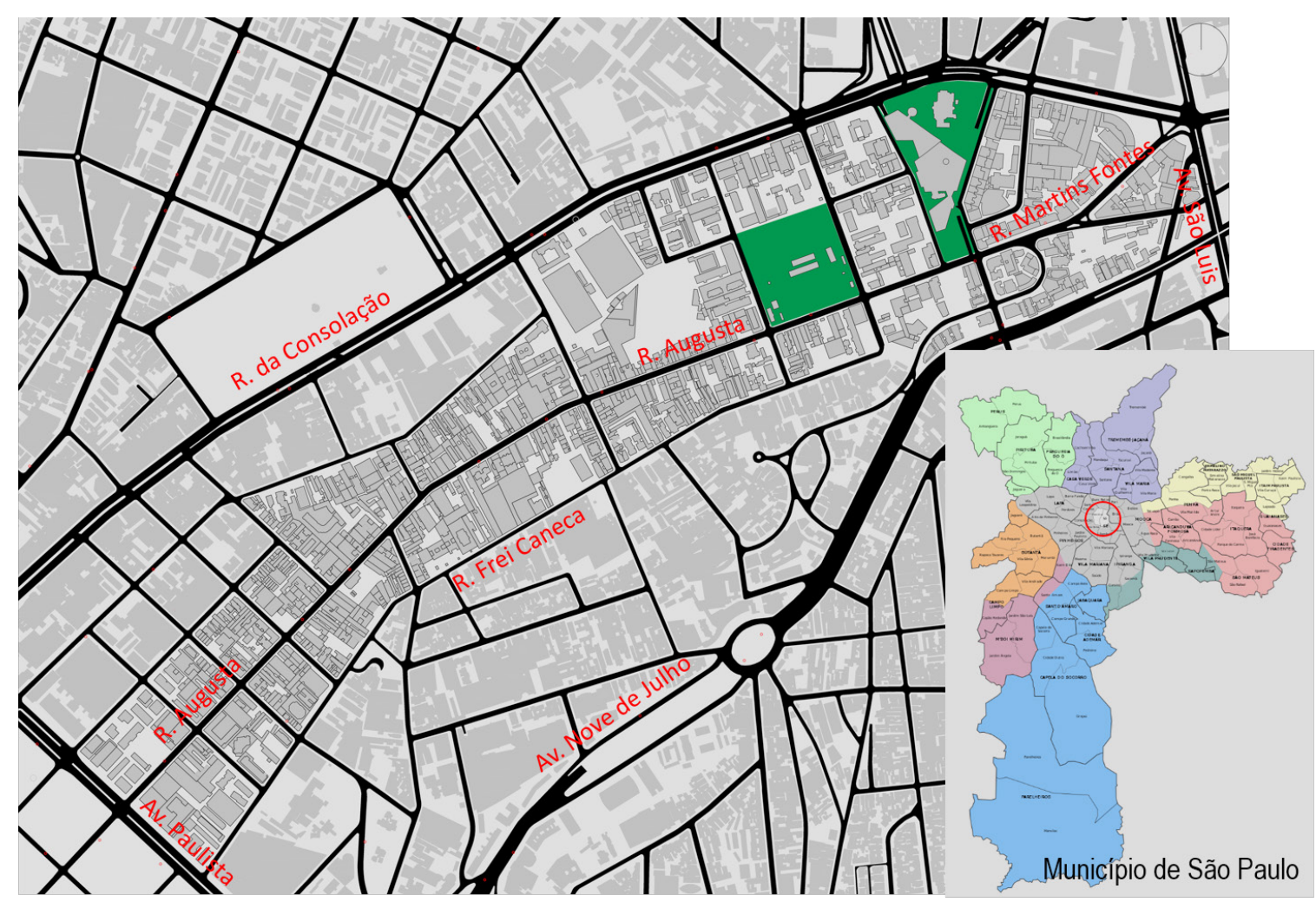

Fig. 1. Localización del área de estudio - Bajo Augusta, Centro de São Paulo. Fuente: Rafael Loddi, 2018 
Y de acuerdo PISSARDO (2012), "en las décadas de 1980 y 1990 , la calle gana fuerza en el arte y los medios al reflejar la crisis económica y social que el país estaba pasando, contrastando con su antigua imagen de prosperidad económica y alto lujo. La constante asociación de una calle al universo del submundo y de la prostitución en los medios y en representaciones artísticas ayudaron a dar una gran exposición al deterioro e ilegalidad de las actividades locales.

Entre 1990 y 2001, de acuerdo con datos de la Secretaría de Planificación, los distritos centrales, Sé y República, perdieron cerca de 20 mil habitantes, pasando de 82 mil a cerca de 62 mil habitantes, resultado en espacios públicos degradados $\mathrm{y}$, marginados, evidenciando el abandono del poder público y una fuerte especulación inmobiliaria.

Hoy, la calle Augusta es un polo multicultural y de ocio nocturno sin incentivos públicos, y con una fuerte especulación inmobiliaria, que puede ser fácilmente comprendida a partir de su evolución histórica.

\section{Objetivo}

El objetivo de este trabajo es recalificar el paisaje urbano y los espacios públicos del Bajo Augusta, desarrollando un núcleo compacto $\mathrm{y}$ diversificado, con conexiones vivas entre personas, lugares y actividades, que fortalezcan la historia y la cultura local, de modo articulado a una movilidad sostenible.

\section{Metodología}

El estudio de caso será en el Bajo Augusta (Figura 1), nombre dado el zona donde pasa la calle Augusta, que conecta la Avenida Paulista a la Plaza Roosevelt sentido centro de São Paulo, teniendo como límite al norte, la Rua da Consolação y al sur, la calle Frei Caneca, en un área de 24,6 ha y 57,138 habitantes (IBGE, 2010).

El análisis de la recalificación urbana del Bajo Augusta se basa en 5 ejes estructuradores: AC - accesibilidad urbana y uso público: crear espacios públicos humanizados y hacer que el área accesible a todos los ciudadanos con diferentes equipamientos públicos y actividades culturales y de ocio; MO - movilidad urbana sostenible: ofrecer conexiones intermodales - ciclista, peatonal y transporte público, y promover el uso de movilidad sostenible - el VLT; IC - identidad cultural: dar énfasis al potencial histórico y cultural local, conectando la cultura con la sociedad y valorizando el patrimonio material e inmaterial; $\mathrm{HB}$ - vivienda social / inclusión social / empleo: aumentar el número de vivienda social en el área central $\mathrm{y}$ incentivar el retrofit en las edificaciones desocupadas o infrautilizadas a lo largo de la calle Augusta; PA - preservación ambiental: ofrecer más calidad de vida, más espacios verdes y mayor área de drenaje natural, con el uso de las tipologías de infraestructura verde.

Se adoptó como premisa los incentivos previstos en el Plan Director Estratégico Ley 16.050 / 2014 - fachada activa, fruición pública, edificios de usos mixtos, aceras anchas y más personas que viven cerca del transporte público; la zonificación vigente - área de Estructuración de la Transformación Urbana, y los instrumentos urbanísticos del Estatuto de la Ciudad - Ley Federal 10.257 / 2001, para ofrecer mayor número de viviendas y mayor oferta de producción artística y cultural con alianzas públicas-privadas.

El barrio posee una densidad demográfica de cerca de 10.000 hab / $\mathrm{km}^{2}$ (IBGE, 2010) - resultado de la verticalización de la región. El área de estudio se concentra en el eje de estructuración y transformación urbana de la calle Augusta, colaborando para una recalificación del paisaje. Las edificaciones tienen cerca de 10 suelos por unidad, con predominio de uso mixto - comercio, servicio y residencia. Poca área verde y arborización en el recorrido de la calle Augusta, concentrando una masa verde en el Parque Augusta. Diagnosticó la existencia de muchas áreas subutilizadas con estacionamientos, los vacíos urbanos.

El transporte urbano se realiza sólo por medio de líneas de autobús que circulan por la calle Augusta, conectando la Av. Paulista al centro ya otros barrios. Se percibe la ausencia de ciclofijas e interconexión entre diferentes modales - autobuses, metro, bicicleta y caminar; y la falta de recorridos entre las pistas para peatones. Se analizó la existencia de grandes cuadras a partir de la tercera cuadra de 


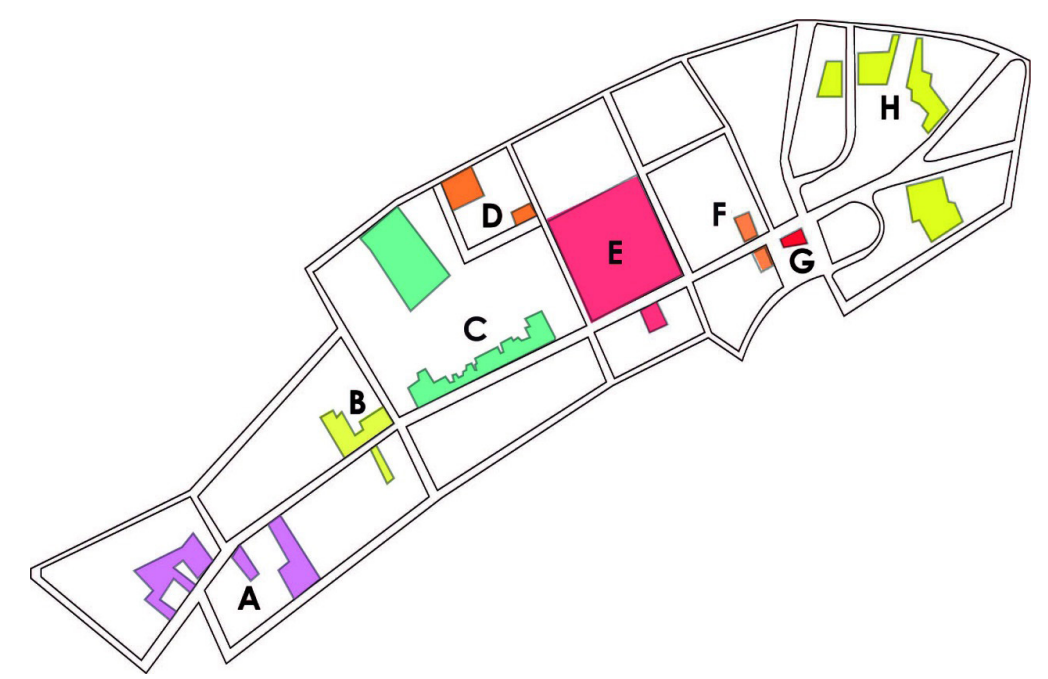

Fig. 2. Sector de la propuesta de intervención. Fuente: Rafael Loddi, 2018

la Avenida Paulista en sentido Centro (a partir de la Av. Fernando de Albuquerque), cuadras con área de 4 ha, siendo el normal cuadras de 1 ha, dificultando la transposición del peatón entre vías y la fruición pública, como la falta de conexión entre la calle Consolação (metro Mackenzie) y la calle Augusta. El flujo de la calle Augusta es caótico con circulación de transporte público, automóviles y peatones en las calles y aceras.

\section{Directrices - propuesta de intervención}

La propuesta de intervención fue dividida en 8 sectores (Figura 2) teniendo en cuenta la necesidad de transformación de los espacios ociosos, la valorización del eje de la calle Augusta y la potenciación de la economía, de la cultura y de la sociedad del Bajo Augusta. La creación de marcos en el paisaje urbano, ofrecerá nuevas centrales y enfatizar la necesidad de los espacios públicos en el eje de la calle Augusta: sector A - Definición de coworking con el objetivo de producir más empleo y renta; y áreas de convivencia pública como food park, cafés, bares, mercadão, parklets, bicicletarios, fortaleciendo el uso de la calle por el peatón; las viviendas sociales para la inclusión social y la reducción de la especulación inmobiliaria; sector B - Nuevas galerías con el objetivo de valorar la historia y la cultura de la calle Augusta, y revitalizar las existentes; y la creación de nuevos parklets; sector C - Galerías subterráneas conectando la calle Augusta a la calle Consolação y propuesta de un nuevo parque público - "Parque das Aguas" instalado en terreno de la SABESP (Compañía de Saneamiento Básico del Estado de São Paulo), con incentivo privado de la misma; sector D - Talleres de cine y teatro valorizando los antiguos caseríos y la cultura local; Townhouse 01 - Taller de Cine "Urban Quilombo" casa grande 02 - Taller de Teatro "Casa Grande Ouro Preto" sector E - Creación del Pabellón cultural y del centro cultural en el Parque Augusta, cuyo objetivo es exponer y divulgar el talento de los artistas de la región. El Parque Augusta fue revitalizado y el área boscosa preservada e insertado nuevos equipamientos urbanos y espacios de ocio y recreación; la industria creativa tendrá el objetivo de producir artesanías y demás confecciones locales, que serán expuestas en el pabellón cultural, estimulando la identidad cultural y colaborando con el aumento de los empleos; sector F - El espacio de convivencia fue proyectado como un pórtico que abraza la calle Augusta y crea espacios de ocio y de interacción con el público - restaurantes, bares, salas interactivas y de exposiciones; sector $\mathrm{G}$ - El Mirador - ubicado en el eje de la radial este (Plaza Roosevelt) con la Rua Augusta, fue proyectado para la producción de atracciones culturales, como un marco de la Rua Augusta; sector H - Las viviendas sociales con áreas de uso común - guardería, cuadras, parque y 
muebles urbanos, proyectadas con diferentes tipologías - 2, 4 y 5 personas / vivienda.

Transformación urbana articulada a la movilidad sostenible

La propuesta de la movilidad se basó en la producción de diferentes modales - vehículo ligero sobre rieles - VLT, metro, bicicleta y auto local o carga y descarga, conectados e integrados a los espacios públicos del Bajo Augusta.

La implantación del VLT (Figura 3), con 5 diferentes líneas conectadas a los principales ejes de expansión de la zona sur y centro, viene como respuesta al incentivo del transporte público en la zona de estructuración urbana (ZEU) y relectura del antiguo tranvía eléctrico de la ciudad de São Paulo, implantadas por la Light a partir de 1900, y asumidas por la Compañía Municipal de Transportes Colectivos (CMTC), que las extinguió a partir de finales de los años 1950.

El eje de la calle Augusta fue segregado entre el paseo público, la vía de autos locales, el VLT con diversas líneas y la ciclovía, incentivando la fruición pública y los espacios del peatón, eliminando el flujo de los automóviles y de los autobuses. El propósito de este cambio fue reducir la contaminación de la calle, extender las aceras y potenciar la movilidad sostenible y la identidad cultural.

La ciclovía fue idealizada a lo largo de la calle Augusta con soporte de bicicletarios en puntos estratégicos - Coworking, Parklets, Parque Augusta, Plaza Roosevelt-Mirante y habitaciones sociales del área central.

El acceso a la estación más cercana de metro - Estación Makenzie, se hizo por galerías subterráneas hasta la Rua da Consolação y por acceso peatonal por la Rua Visconde de Ouro Preto. Los espacios ociosos, de estacionamiento, sufrieron nuevos usos de acuerdo con la necesidad local - viviendas sociales, galerías, parklets y foodparks (Figura $4)$.

\section{Adensamiento constructivo y habitacional}

El aumento del potencial constructivo combinado con la definición de la Cota Parte Máxima de Terreno por Unidad induce el adensamiento constructivo y habitacional de

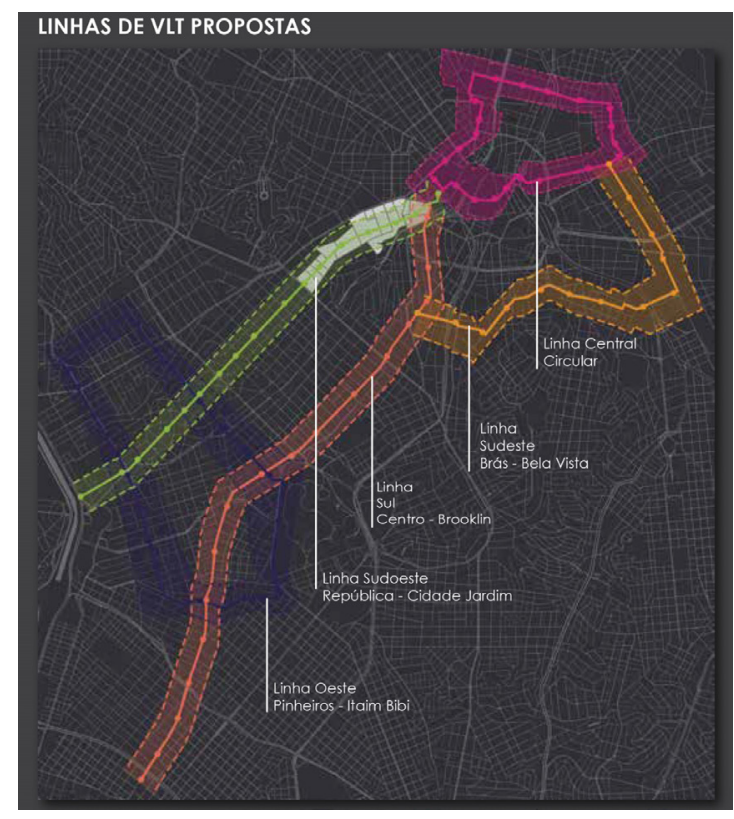

Fig. 3. Propuesta de líneas de VLT.

Fuente: Rafael Loddi, 2018

estas áreas, optimizando el uso de la tierra en áreas bien ubicadas.

El área de intervención insertada en la Zona Eje de Transformación Urbana (ZEU) y en la Zona Centralidad (ZC), posibilitó la producción de un mayor número de vivienda social en terrenos ocupados con estacionamientos, proporcionando núcleos compactos con adensamiento, verticalización y nuevas tipologías con diferentes densidades. La intención es transformar un área con alto grado de especulación inmobiliaria en espacios orientados a la inclusión de la sociedad. Las edificaciones en mal estado de conservación fueron revitalizadas, y los lotes sin uso apropiado, sufrieron nuevos usos, coherentes con la necesidad local y con las dinámicas estructuradoras de la propuesta.

\section{Corredor verde - Calle Augusta}

La calle Augusta tendrá la función de corredor verde con el uso de las tipologías de infraestructura verde, promoviendo hábitats para fauna y flora, drenaje natural del sistema viario para la cuenca del arroyo Augusta, y un equilibrio estético y paisajístico: la laguna pluvial proyectada en el Parque Augusta tendrá la función de retención de agua de lluvia para 
reutilización en la irrigación de la arborización y de los canteros del parque; el techo verde estará presente en los edificios de Vivienda Social con la función de absorber el agua de lluvia, reducir el efecto de la isla de calor urbano, contribuir a la eficiencia energética de las edificaciones y crear hábitat para vida silvestre; los jardines de lluvia fueron proyectados en la calle Augusta, en el eje tomado por el lecho del VLT, ya con depresión topográfica natural, para recibir el flujo del agua pluvial proveniente de áreas impermeables y para recibir el flujo superficial que carga los contaminantes del paseo público, creando un ambiente más atractivo y seguro para los peatones; las biovaletas, fueron proyectadas en los canteros de la calle Augusta, llenas de vegetación, suelo y demás elementos filtrantes, que procesan una limpieza del agua de lluvia, al mismo tiempo que aumentan su tiempo de flujo, dirigiéndolo a los jardines de lluvia central.

La infraestructura verde renovará el espacio público de la calle Augusta como un elemento vital a la ciudad, colaborando en su revitalización, tanto ecológica como social y económica. También tendrá como función la filtración del aire y del agua, y proporcionará a la población espacios de ocio y educación ambiental. Se trata de una infraestructura que promoverá la biodiversidad en el entorno urbano y proporcionará una sostenibilidad ambiental, esencial para preservar los recursos naturales que sustentan la ciudad y la sociedad.
El corredor verde es la conexión viva y la identificación de los peatones con la calle y la ciudad, conectando las diferentes redes proyectadas en el área de intervención; desde la red de movilidad y sus diferentes modales - VLT, bicicletas, peatones, etc; la red de accesibilidad urbana - ofreciendo caminos y tramos sombreados y placenteros al peatón, la red ecológica - ofreciendo un corredor ecológico a la fauna y flora, y la malla cultural - creando recorridos entre las diversas actividades culturales (cines, teatros, talleres, espacio cultural con comodidad y conveniencia), y colaborando con la preservación del patrimonio histórico y cultural.

\section{Conclusión}

En vista de la importancia de la calle Augusta en la historia de la ciudad de São Paulo y la actual situación en que se encuentra, la propuesta pone de manifiesto la necesidad de establecer conexiones sostenibles en diferentes escalas urbanas, nuevas oportunidades de trabajo, ocio y cultura, inclusión social, preservación ambiental y cultural y una movilidad urbana compatible con la realidad local. Podemos concluir que la propuesta de intervención colaborará con el proyecto de recalificación del área central de la ciudad por los organismos públicos, promoviendo calidad de vida y calidad ambiental urbana, y trae una nueva percepción de la forma de apropiación

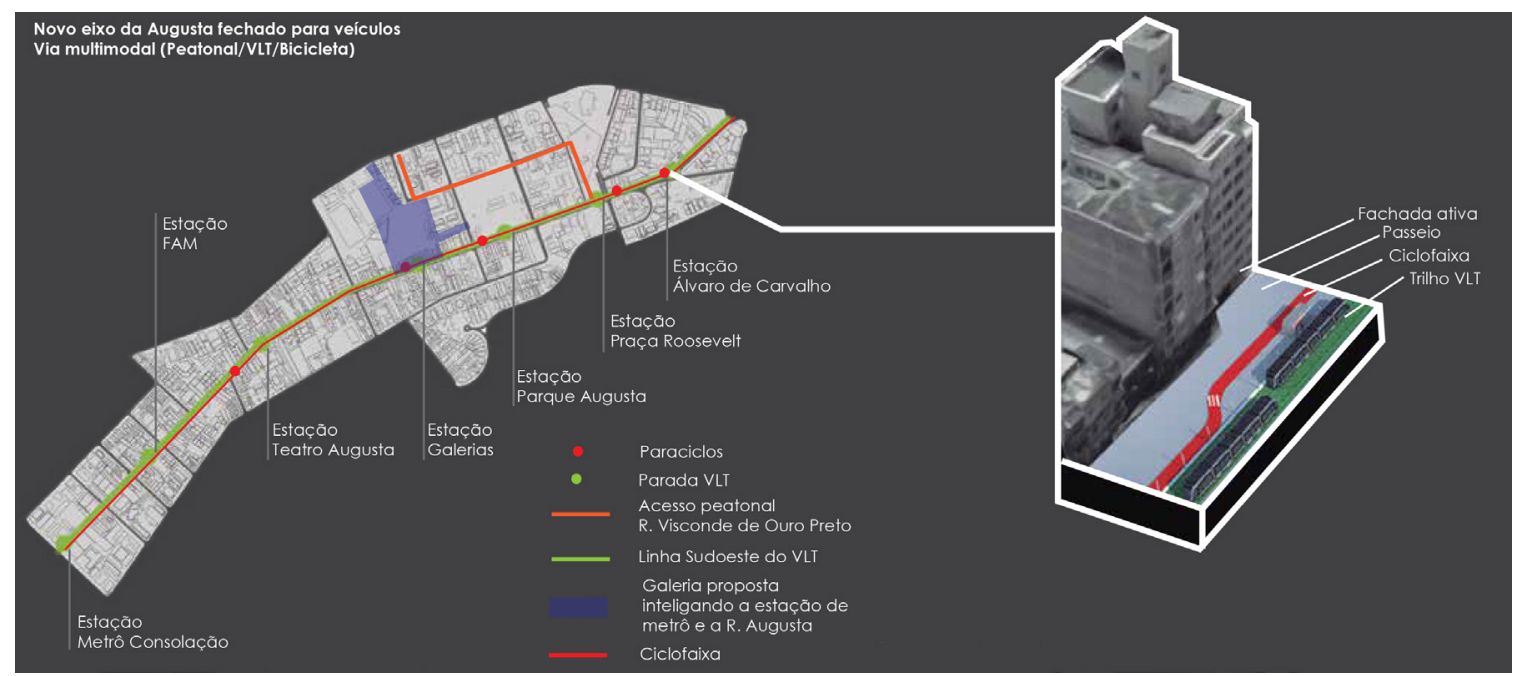

Fig. 4. Propuesta de movilidad sostenible. Fuente: Rafael Loddi, 2018 
del espacio urbano, de valorización de la identidad cultural.

\section{Referencias}

Ahern, J. 2002. Greenways as Strategic Landscape Planning: theory and application. Wageningen University, Wageningen.

Benedict, Mark A., and Edward T. McMahon. 2006. Green Infrastructure: Linking Landscapes and Communities. Island Press

Brasil. (2001) Lei 10.257 de 10 de julho de 2001: Regulamenta os artigos 182 e 183 da Constituição Federal, estabelece diretrizes gerais da política urbana e dá outras providências. Brasília.

(2014) Lei 16.050, de 31 de julho de 2014: Institui o Plano Diretor Estratégico e o Sistema de Planejamento e Gestão do Desenvolvimento Urbano do Município de São Paulo. São Paulo: Prefeitura do Município de São Paulo.

Cano, Wilson. Desequilíbrios regionais e concentração industrial no Brasil 19301970. São Paulo. Universidade Estadual de São Paulo, 2007.

EMURB, Caminhos para o Centro: Estratégias de Desenvolvimento para a Região Central de São Paulo. São Paulo, 2004. p. 363-379

O Estado de São Paulo. (1987) "Candeias e as Belas da Billings”. São Paulo. 13/02/1987, Página 3. Caderno 2.

O'neil, Alistair. 2002. John Stephen: uma apresentação de masculidade da Carnaby Street 1957-75. Fashion Theory. Vol I, No 4 , p-119-138.

Pissardo, Felipe M. A rua apropriada: um estudo sobre as transformações e usos urbanos na Rua Augusta. (São Paulo 18912012). São Paulo, 2013.

Ragazzo, Cleber. 2005. Rua Augusta: A calçada da glória. São Paulo: Digerati Books.

Rogers, Richard; Gumuchdjian, Philip. 1997. Cidades para um Pequeno Planeta. Barcelona. Editora Gustavo Gili.

Rolnik, Raquel; Klintowitz, Danielle. 2011. (I) Mobilidade na cidade de São Paulo.

Sandroni, Paulo. A dinâmica imobiliária da cidade de São Paulo: esvaziamento, desvalorização e recuperação da região central. In EMURB, 2017.
Villaça, Flávio. 2001. Espaço intra-urbano no Brasil. São Paulo: Studio Nobel $2^{a}$ Edição.

Zimmermann, Maíra. Rua Augusta: Juventude e Sociabilidade no Espaço Urbano, (196873). Anais do XXVI Simpósio Nacional de História - ANPUH, São Paulo, 2011. 